\title{
AUFWACHSEN IN STATIONÄRER JUGENDHILFE UND DIE BEDEUTUNG FÜR DEN BIOGRAFISCHEN VERLAUF BETROFFENER JUNGER MENSCHEN - EINE EMPIRI- SCHE LÄNGSSCHNITTANALYSE ZUM SOZIALISATI- ONSKONTEXT HEIMERZIEHUNG
}

\author{
Alexander Parchow und Tim Middendorf \\ Fachhochschule Münster, Fachbereich Sozialwesen \\ E-Mail: alexander.parchow@fh-muenster.de \\ URL: https://www.fh-muenster.de/sw/personen/Wissenschaftliche_Mitarbeiterln- \\ $\underline{\text { nen.php?pld=8976\&orga }=10 \& m \_l i s t \_i d=0 \& p \_l i s t \_i d=20}$ \\ Katholische Hochschule NRW, Abteilung Münster \\ E-Mail: t.middendorf@katho-nrw.de \\ URL: https://www.katho-nrw.de/muenster/studium-lehre/lehrende/wissenschaftlich- \\ mitarbeitende/middendorf-tim-dr-phil/
}

Zitationsvorschlag:

Parchow, Alexander/Middendorf, Tim (2021): Aufwachsen in stationärer Jugendhilfe und die Bedeutung für den biografischen Verlauf betroffener junger Menschen - eine empirische Längsschnittanalyse zum Sozialisationskontext Heimerziehung. In: Gesellschaft - Individuum - Sozialisation (GISo). Zeitschrift für Sozialisationsforschung, 2 (2).

DOI: 10.26043/GISo.2021.2.2

Link zum Artikel:

https://doi.org/10.26043/GISo.2021.2.2 


\title{
AUFWACHSEN IN STATIONÄRER JUGENDHILFE UND DIE BEDEUTUNG FÜR DEN BIOGRAFISCHEN VERLAUF BETROFFENER JUNGER MENSCHEN - EINE EMPIRI- SCHE LÄNGSSCHNITTANALYSE ZUM SOZIALISATI- ONSKONTEXT HEIMERZIEHUNG
}

\author{
Alexander Parchow und Tim Middendorf
}

Die selbst- oder fremdbestimmte Unterbringung von Kindern und Jugendlichen in einer Maßnahme der stationären Erziehungshilfe stellt einen starken Eingriff in deren biografischen Verlauf dar. Über die langfristigen (sozialisatorischen) Auswirkungen des Aufwachsens in Einrichtungen der Heimerziehung sind nur wenige Erkenntnisse vorhanden.

In diesem Artikel gehen wir anhand einer Einzelfallanalyse der Frage nach, welche Sozialisationseffekte aus dem Lebensereignis Heimerziehung emergieren und wie sie im weiteren biografischen Verlauf von Adressat*innen sichtbar werden. Das längsschnittliche Datenmaterial basiert auf fünf Interviewerhebungen über einen Gesamtzeitraum von zehn Jahren während und nach dem Aufenthalt in Einrichtungen der stationären Jugendhilfe. Wir identifizieren und analysieren emergierende Sozialisationseffekte in ihrer Bedeutung für den weiteren biografischen Verlauf entlang der drei Analysedimensionen Wohnen, soziale Beziehungen und beruflicher Werdegang. Dabei wird die Bedeutung eines verlässlichen Beziehungsangebots pädagogischer Fachkräfte sowie eines auf die individuellen Bedürfnisse von fremduntergebrachten jungen Menschen abgestimmten professionellen Handelns sichtbar.

Keywords: Jugendhilfe, stationäre Erziehungshilfe, Heimerziehung, Sozialisation, Längsschnitt

\section{EINLEITUNG}

Für alle Beteiligten, allen voran für die fremdplatzierten Menschen, stellt die Fremdunterbringung in einer Einrichtung der stationären Erziehungshilfe ein zu bewältigendes „kritisches Lebensereignis" (Lambers 1996) dar. Bisher sind ein Großteil der empirischen Studien im Feld der Heimerziehungsforschung auf die Effekte, den Erfolg der Maßnahme sowie die Wirkungen auf ihre Adressat*innen während oder unmittelbar nach der Hilfe beschränkt. In den letzten Jahren wurde zwar die Forschungsperspektive zunehmend um den (gelingenden) Übergang von der Jungendhilfe in ein eigenständiges Leben erweitert, jedoch erstrecken sich diese Untersuchungszeiträume vorwiegend über die Zeit kurz nach der Heimerziehung (Strahl 2020, 24). Somit ist ein Forschungsdesiderat einerseits über die biografischen Verläufe und Lebenslagen junger Menschen lange nach Verlassen der Jugendhilfe sowie andererseits über die langfristigen Effekte stationärer Erziehungshilfe zu konstatieren.

Für entsprechende Untersuchungen drängt sich eine sozialisationstheoretische Perspektive auf. Das kritische Lebensereignis Heimunterbringung wird durch diese Betrachtungsweise als ein Sozialisationskontext gesehen, der sich mit weiteren Sozialisationsprozessen der jungen Menschen interdependent verwebt. So werden wechselseitige Vermittlungsprozesse von Individuum und Gesellschaft sichtbar - ein Kernthema von Sozialisation (Abels 2015, 50).

In diesem Artikel beschäftigen wir uns deshalb aus einer sozialisationstheoretischen Perspektive mit der Bedeutung des Aufwachsens in stationärer Jugendhilfe für den weiteren biografischen Verlauf. Genauer gesagt fragen wir danach, welche Sozialisationseffekte aus dem Lebensereignis Heimerziehung emergieren 
und wie sie im weiteren biografischen Verlauf von Adressat*innen sichtbar werden.

Unter dem Begriff biografischer Verlauf verstehen wir in unserem Beitrag eine individuelle und ganz persönliche Lebensgeschichte, die durch ihre soziale Determiniertheit gesellschaftliche Strukturen wiederspiegelt (Lamnek/Krell 2016, 633 f.). In diesem Sinne analysieren wir das Interviewmaterial einer jungen Frau mit Unterbringungserfahrung aus einer Längsschnittstudie.

Der Artikel ist wie folgt gegliedert: Einführend wird im zweiten Kapitel der Forschungsstand anhand themenrelevanter Studien skizziert, bevor ein Einblick in den Entstehungskontext des längsschnittlich erhobenen Datenmaterials gewährt wird. Daran schließt sich in Kapitel vier die Darstellung der sozialisationstheoretischen Perspektive unseres Analysefokus an, um im fünften Kapitel das forschungsmethodische Vorgehen der Analyse für diesen Beitrag zu explizieren. Es folgt im sechsten und siebten Kapitel die Ergebnisdarstellung: Zunächst wird der biografische Verlauf entlang der verschiedenen Erhebungszeitpunkte expliziert, um inn anschließend sozialisationstheoretisch zu analysieren. Zentrale Punkte werden in einem Resümee zusammengefasst.

\section{FORSCHUNGSSTAND ZUM SOZIALISATI- ONSKONTEXT HEIMERZIEHUNG}

Innerhalb der Heimerziehungsforschung liegt mittlerweile eine Vielzahl unterschiedlicher Studien vor, die kaum vollständig und systematisch zu erfassen sind (Nüsken/Böttcher 2018, 26). Für diesen Beitrag liegt der Fokus der nachfolgenden Darstellung auf der systematischen Erfassung sozialisatorisch relevanter Erkenntnisse im Kontext Heimerziehung.

Seit den 1990er-Jahren tauchten im Forschungsfeld der Heimerziehung vermehrt biografisch angelegte Untersuchungen auf, die häufig darauf abzielten, Wirkfaktoren gelingender Heimerziehung sowie die Bedeutung der Heimerziehung für den biografischen Verlauf fremduntergebrachter junger Menschen zu erkunden (beispielsweise Lambers 1996; Gehres 1997; Normann 2003; Finkel 2004; Rätz-Heinisch 2005).
Lambers merkt an, dass die Unterbringung in stationärer Hilfe ein kritisches Lebensereignis im biografischen Verlauf für die Betroffenen darstellt, dessen Bewältigung von Seiten der Jugendhilfe unterstützt werden muss (Lambers 1996, 50). Die Wahrscheinlichkeit einer positiven Bewältigung wird insbesondere dadurch erhöht, dass die stationäre Maßnahme an die sozialisatorischen Bedingungen des Aufwachsens, die hier erworbenen Kompetenzen und Handlungsmuster sowie die biografischen Erfahrungen anschlussfähig ist (Rätz-Heinisch 2005, 299; Finkel 2004, 314). Laut Finkel muss die Maßnahme neben dieser (biografischen) Passung dem Bedürfnis nach Geborgenheit, Selbständigkeit und Eigensinn entsprechen, um positive sozialisatorische Wirkungen bei den jungen Menschen entfalten zu können (Finkel 2004, 309). Die Herstellung einer Passung liegt so gesehen in besonderem Maß bei den am Hilfeprozess beteiligten Fachkräften, indem sie die jungen Menschen u. a. an Entscheidungen partizipieren lassen, den Übergang in die Einrichtung intensiv vorbereiten und gestalten sowie auf den Erhalt von Beziehungen zu Personen außerhalb der Maßnahme bedacht sind (Macsenaere/Esser 2015, 51).

Darüber hinaus weisen vor allem Wieland et al. auf die Notwendigkeit einer vertrauensvollen Beziehung zwischen Fachkräften und fremduntergebrachten jungen Menschen hin (Wieland et al. 1992, 12), die, wie auch Rätz-Heinisch feststellt, den jungen Menschen verlässlich und über eine längere Zeit hinweg beständig angeboten werden sollte (Rätz-Heinisch 2005, 315).

Unter Berücksichtigung der genannten Faktoren ergibt sich die Chance, dass das kritische Lebensereignis Heimunterbringung retrospektiv als Unterstützung im weiteren biografischen Verlauf betroffener junger Menschen angesehen werden kann (Normann 2003, 155).

Seit einigen Jahren wurde der wissenschaftliche Diskurs zur Heimerziehung um die Phase des Übergangs vom Jugendhilfesystem in ein eigenständiges Leben erweitert. Für diesen als Leaving Care bezeichneten Prozess formulieren Hansbauer und Kress Gelingensfaktoren (Beziehungsgestaltung zur Herkunftsfamilie, Freunden und den Fachkräften, Entwicklung von Sozial- 
und Selbstkompetenz sowie berufliche Integration), die im Übergang von der stationären Maßnahme in die Selbständigkeit dienlich sind (Hansbauer/Kress 2012, 10).

Die SOS-Längsschnittstudie besitzt einen anderen und für den Beitrag ebenfalls relevanten Fokus: Sie untersucht die Entwicklung von Handlungsbefähigung durch das Aufwachsen in Heimerziehung als ein Zusammenspiel von Resilienz, Selbstwirksamkeit und Salutogenese. Zudem geht sie der Frage nach, wie die Handlungsbefähigung durch pädagogische Fachkräfte gefördert werden kann, damit sie den jungen Menschen als Sozialisationseffekt im Care-Leaving-Prozess als Metaressource zur Verfügung steht (Sierwald et al. 2017, 11 f.). In ersten Zwischenergebnissen konnte $u$. a. festgestellt werden, dass die Handlungsbefähigung dann zunimmt, wenn die jungen Menschen in hohem Maße an Entscheidungen partizipieren sowie Anerkennung und Wertschätzung durch Fachkräfte erhalten (ebd., 15).

Insgesamt ist zu konstatieren, dass langfristige sozialisatorische Wirkeffekte über den Zeitraum der Heimunterbringung hinaus durch den aktuellen Forschungsstand kaum in den Blick genommen werden. Denn sozialisationstheoretische Forschungen profitieren vor allem von längsschnittlich erhobenen Daten, die mithin kaum vorliegen oder nur einen kurzen Zeitraum umfassen.

Gegenwärtig sind innerhalb der Heimerziehungsforschung lediglich zwei über einen längeren Zeitraum verlaufende Längsschnittstudien im deutschsprachigen Raum relevant, in denen auch (lange) nach der Heimunterbringung Daten erhoben werden: die schon benannte, im Jahr 2014 begonnene SOS-Längsschnittstudie sowie die Münsteraner Längsschnittstudie.

Die empirische Grundlage dieses Beitrags entstammt den Daten der Münsteraner Längsschnittstudie. Unsere Analyse umfasst die Zeit der Unterbringung, die Leaving-Care-Phase sowie das Leben nach der Jugendhilfe. Durch den langen Zeitraum geraten Sozialisationseffekte über die Grenzen der Heimerziehung und des Leaving Care in den Blick, um das Lebensereignis Heimerziehung im biografischen Verlauf zu verorten.

\section{DIE MÜNSTERANER LÄNGSSCHNITTSTU- DIE}

Im Jahr 2008 initiierte Prof. Dr. Peter Hansbauer von der Fachhochschule Münster in Kooperation mit der Diakonie Rheinland-Westfalen-Lippe und finanzieller Unterstützung seitens der FazitStiftung sowie des damaligen Ministeriums für Generationen, Familie, Frauen und Integration des Landes Nordrhein-Westfalen eine prospektive Längsschnittstudie mit dem Ziel, die Folgen und die subjektive Relevanz von stationärer Heimunterbringung im Leben betroffener junger Menschen zu untersuchen. Die Studie war auf eine Dauer von zehn Jahren mit fünf Erhebungszeitpunkten angelegt. ${ }^{1}$ Zum Zeitpunkt der ersten Erhebung nahmen 102 junge Menschen aus 65 unterschiedlichen Maßnahmen von 17 unterschiedlichen Trägern teil, die ein Mindestalter von 15 Jahren aufwiesen und sich in einer Maßnahme der Heimerziehung befanden. Die Teilnehmenden wurden in regelmäßigen Abständen ca. alle zwei Jahre während der Fremdunterbringung, im Übergang von der stationären Maßnahme in die Eigenständigkeit und (lange) nach der Jugendhilfezeit befragt. An allen fünf Erhebungszeitpunkten wurden drei Instrumente eingesetzt: erstens ein standardisierter Fragebogen zur Ermittlung der soziodemografischen Merkmale sowie der Norm- und Wertvorstellungen mittels Itembatterien aus der Shell Jugendstudie und dem Sozio-oekonomischen Panel; zweitens eine standardisierte ego-zentrierte Netzwerkkarte zur Erhebung der Beziehungskonstellationen und der Eingebundenheit in soziale Netzwerke; und drittens ein Leitfadeninterview zur Erhebung vergangener subjektiv wichtiger Erfahrungen und zur Ermittlung der (retrospektiven) Sicht auf verschiedene Aspekte der Heimunterbringung (Parchow 2021, 117).

Die vorerst letzte Erhebung fand Anfang 2019 statt, die Auswertung der Daten ist gegenwärtig

\footnotetext{
${ }^{1}$ Die verschiedenen Erhebungszeitpunkte sind im weiteren Textverlauf mit T1 für Zeitpunkt 1, T2 für Zeitpunkt 2 usw. gekennzeichnet
} 
noch nicht abgeschlossen. Insofern ist ein Rückbezug der hier dargestellten Einzelfallanalyse auf die Gesamtergebnisse der Studie noch nicht möglich.

\section{EINE SOZIALISATIONSTHEORETISCHE PERSPEKTIVE AUF HEIMERZIEHUNG}

Aus einer sozialisationstheoretischen Perspektive gehen wir in unserer Einzelfallanalyse der Frage nach, welche Hinweise auf Sozialisationsgeschehen in Heimerziehung zu identifizieren sind. Folgen wir der grundlegenden Definition von Sozialisation als „to make fit for living in society“" (Clausen 1968, 21), dann impliziert Sozialisation sowohl Prozesse von Individualgenese und Handlungsbefähigung als auch von gesellschaftlicher Anpassung und Eingliederung (vgl. Grundmann 2006, 17).

Diese Prozesse sind im Kontext der stationären Jugendhilfe grundlegend zu achten und zu fördern: „Jeder junge Mensch hat ein Recht auf Förderung seiner Entwicklung und auf Erziehung zu einer eigenverantwortlichen und gemeinschaftsfähigen Persönlichkeit“" ( 1 Abs. 1 SGB VIII). Aus diesem Blickwinkel kann Heimerziehung als ein begleitetes Hineinwachsen in differente Gemeinschaften bei gleichzeitiger Individualgenese und Handlungsbefähigung von Adressat*innen betrachtet werden. Das Hineinwachsen in gesellschaftliche Strukturen umfasst zudem deren interaktionale Gestaltung und Prägung durch die Adressat*innen. Da sich Sozialisation im Spektrum von Vergesellschaftung und Individuation ereignet (Bauer 2012, 474), fassen wir das Aufwachsen in stationärer Jugendhilfe als Sozialisationskontext.

Der Vollzug stationärer Jugendhilfe ist demnach ein komplexes und herausforderndes Sozialisationsgeschehen. Abseits der vielfach defizitären Entwicklungsvoraussetzungen der Adressat*innen durch ihre primären Sozialisationserfahrungen in der Herkunftsfamilie (Fendrich et al. 2018, 21) birgt die außerfamiliäre Unterbringung gleichzeitige Anpassungs- und Eingliederungsanforderungen: Die Adressat*innen sind aufgefordert, sich in spezifische Strukturen der stationären Einrichtung - die oft abseits des Herkunftsmilieus verortet sind - und zugleich in gesamtgesellschaftliche Prozesse - die ihr erweitertes soziales Umfeld umfassen - zu integrieren. Die Erfahrungsbiografie der Adressat*innen, gepaart mit determinierenden Kontextfaktoren auf der Meso- (z. B. Institutionen und Träger) und Makroebene (z. B. gesellschaftliche Erwartungen, Werte und Normen), und die mikrosozialen Bezugnahmen mit verschiedenen Beteiligten unterschiedlicher Funktionsbereiche (z. B. Jugendhilfeeinrichtung und Jugendamt) führen zu einem mehrdimensional verwobenen Sozialisationsgeschehen.

Zur Erfassung und Analyse des komplexen Sozialisationszusammenhangs Heimerziehung bietet das allgemeine "Modell von Sozialisation als soziale Praxis der Hervorbringung von Personalität und Sozialität" (Grundmann 2006, 47) ein angemessenes Instrument. Als Idealmodell bietet Grundmann einen besonderen Wert für die empirische Sozialisationsforschung, da das Gelingen von Sozialisation durch Prüfkriterien gemessen werden kann (Abels 2015, 76).

Grundmann fasst als zentrales Kriterium von Sozialisation eine wechselseitige Bezugnahme von Akteur*innen, in welche die Erfahrungsbiografie der Beteiligten als reflexive Subjekte einfließt: Die emergenten Strukturen und inhaltlichen Kriterien der Bezugnahme bringen Interaktionserwartungen und -notwendigkeiten hervor, die wiederrum rekursiv durch die Interaktion der Teilnehmenden als gemeinsamer situativer Handlungsbezug prozessual modifiziert werden. Aus der auf diese Weise ko-konstruktiv hergestellten Sozialisationspraxis emergieren soziale Kompetenzen, Handlungsorientierungen und Handlungsbefähigungen bei den Beteiligten (Grundmann 2006, $46 \mathrm{ff}$.$) .$

In diesem Beitrag werden die mikrosozialen Interaktionen im Kontext Heimerziehung indirekt über die subjektive Sicht der Befragten erfasst, um „die Zusammenhänge zwischen den Persönlichkeitsentwicklungen und den sie prägenden sozialen Umwelten zu untersuchen“ (ebd., 204). Denn wie von Grundmann festgehalten, prägen die sozialisatorischen Interaktionen die sozialen Umwelten und werden durch sie geprägt (ebd., 47 f.). 


\section{METHODISCHES VORGEHEN IN DER EIN- ZELFALLANALYSE}

Für die Einzelfallanalyse zur Bearbeitung unserer Fragestellung wurde aus der Münsteraner Längsschnittstudie ein Fall ausgewählt, zu dem für alle Erhebungszeitpunkte Daten vorliegen und der hohe narrative Erzähleinheiten in den Interviews zur Exploration reflexiver und vorreflexiver Sozialisationsprozesse beinhaltet. ${ }^{2}$

Die Interviews aller fünf Erhebungsschnittpunkte wurden in methodischer Anlehnung an die Grounded Theory-Methodologie nacheinander kodiert (Strauss/Corbin 1996, 43-117). Zur Steigerung der theoretischen Sensibilität (ebd., 25) vollzog sich der Kodierprozess gemeinsam durch beide Verfasser dieses Beitrags in einem verstetigten und diskursiven Austausch. Die induktiv und abduktiv gewonnen Erkenntnisse aus dem empirischen Datenmaterial wurden zirkulär-iterativ mit sensibilisierenden Konzepten aus eigenen Dissertationsprojekten sowie einem weiterführenden Literaturstudium verschränkt. Im Ergebnis entstand ein strukturierendes AnaIyseraster, das zur Gliederung des Falls genutzt wurde.

Zur weiteren Analyse und zur Ergebnispräsentation wurde der Fall in einem zweiten Analyseschritt nah am Datenmaterial und entlang der Erhebungszeitpunkte zusammengefasst, um abschließend vollzogene Sozialisationsprozesse zu identifizieren. Dabei wurden die kodierten Textsegmente innerhalb der Analysedimensionen aus den verschiedenen Interviews im Längsschnitt betrachtet, um insbesondere retrospektiv Neu- bzw. Umbewertungen von Aspekten der Heimzeit zu erkennen.

Die Einzelfallstudie unterliegt forschungsethischen Spannungsfeldern. Das Datenmaterial verschafft einen Einblick in ganz persönliche Lebenswelten und fördert die Zuschreibung als Adressatin der Jugendhilfe. Wir haben uns im Sinne der ,[f]orschungsethische[n] Prinzipien und wissenschaftliche[n] Standards für Forschung der Sozialen Arbeit" (Franz/Unterkofler 2021, 39) für die Aussparung hochsensibler Themenbereiche und persönlicher Netzwerkkarten entschieden (ebd., 51).

\section{VIELE MENSCHEN UND DOCH ALLEIN? - DER FALL SASKIA}

Bei dem vorzustellenden Fall handelt es sich um die junge Frau Saskia ${ }^{3}$. Ihr biografischer Verlauf ist geprägt von vielen Lebensortwechseln, die entlang der verschiedenen Erhebungszeitpunkte als subjektive Perspektive sichtbar werden. Mit neun Jahren wurde sie erstmals aus dem Haushalt ihrer alleinerziehenden Mutter für vier Jahre in einem Wohnheim untergebracht. Nach einem anschließenden Aufenthalt von etwa einem Jahr bei ihrem Vater zog sie erneut in eine stationäre Maßnahme der Jugendhilfe, um nach einem Jahr in ein betreutes Einzelwohnen zu wechseln. Nach weiteren sechs Monaten zog Saskia zunächst zu ihrer Mutter und im Anschluss zu ihrem Vater, um nach einigen Jahren alleine in eine Wohnung umzuziehen.

\section{1 „Das ist voll viel, was die von einem wollen." ${ }^{4}$ - Die erste Befragung}

Zu Beginn der Studie im Jahr 2008 war Saskia bei der ersten Befragung 15 Jahre alt. Sie lebte zu diesem Zeitpunkt mit anderen jungen Mädchen in einer ausgelagerten Wohngruppe und besuchte die Realschule. Ihre Mitbewohnerinnen beschrieben sie als „lieb und nett“ (Int. T1, Abs. 5) und die Betreuer*innen sah sie als neutrale, stetig verfügbare Ansprechpersonen. Saskia störten vor allem die an sie gestellten Anforderungen: Sie sollte ihr soziales Leben in die Wohngruppe verlagern, zwingend an Gruppenaktivitäten teilnehmen und durfte sich außerhalb des Heimkontextes nur in festen und starren Zeitenräumen bewegen. Zum Thema Freundschaften sagte Saskia: „Ich soll nicht so

\footnotetext{
2 Der Begriff des Falls als Bezeichnung von Adressat*innen in ihrer Eingebundenheit in Lebenswelten ist in der Jugendhilfe gängig. Wir plädieren aus sozialisationstheoretischer Perspektive für einen erweiterten Fallbegriff in Anlehnung an Ader und Schrapper, der Adressat*innen und ihre sozialen Umwelten inklusive der Helfer*innensysteme einschließt (Ader/Schrapper 2020, 36-40).

${ }^{3}$ Zur Wahrung der Anonymität wurde der Namen geändert. Zudem verzichten wir auf die Benennung von Orts- und Einrichtungsnamen.

${ }^{4}$ Saskia Int. T1, Abs. 7.
} 
viel mit meinen Freunden machen, ich soll die eher ganz hinten hinschieben, aber mehr hier sein" (Int. T1, Abs. 7).

Diese Anforderungen missfielen Saskia und sie verglich ihr gegenwärtiges Handeln mit dem Verhalten in der vorherigen Unterbringung: „Und auch in der anderen Wohngruppe, da hab ich so mein Ding in die Hand genommen. Also ich hab mir nichts einreden lassen und ich hab mich nicht veräppeln lassen“ (Int. T1, Abs. 45).

Als zentrale Lebensereignisse in ihrem biografischen Verlauf benannte Saskia in der ersten Befragung die Scheidung ihrer Eltern und die daraus resultierenden Folgen: „[A]lso ich war eher so auf mich allein gestellt" (Int. T1, Abs 45). Sie bewertete die Situation retrospektiv als einschneidend: „Ja, es war sehr schlimm und traurig, aber dadurch bin ich mehr selbständig geworden“ (Int. T1, Abs. 47). Aus der Trennung ihrer Eltern leitete sie auch den Grund für den Einzug in die erste Wohngruppe im Alter von neun Jahren her: „Dadurch, dass sie im Alkohol versunken ist [die Mutter; Anm. der Verf.], musste ich in ein Heim, weil mein Vater mich nicht wollte wegen seiner neuen Frau" (Int. T1, Abs. 3).

Zwischen der ersten Unterbringung und der Wohngruppe zum Zeitpunkt der ersten Befragung lebte Saskia im Alter von 13 Jahren etwa ein Jahr bei ihrem Vater und seiner neuen Lebensgefährtin, was sie als weiteres wichtiges Lebensereignis ansah:

„[D]ann hat seine Frau sich doch entschieden mich zu mögen, und somit durfte ich dann da raus und konnte dann ein Jahr bei ihnen wohnen. Das hat aber gar nicht mit ihr geklappt. [...] Wir haben nur gestritten. Ja, dann hat mein Vater gesagt, ich möchte meine Lebensgefährtin nicht verlieren, aber Saskia kann dann wieder zurück ins Heim." (Int. T1, Abs. 3)

\section{2 „Ja teilweise fand ich auch, wars schon läs- tern. ${ }^{5}$ - Die zweite Befragung}

Saskia war zum Zeitpunkt der zweiten Datenerhebung 17 Jahre alt. Sie hatte die Jugendhilfe verlassen und wohnte seit zwei Wochen im
Haushalt ihres Vaters und dessen Lebensgefährtin. Saskia ging zu diesem Zeitpunkt weiter zur Schule, um das Abitur zu erreichen.

Saskia berichtete retrospektiv über die Zeit nach der ersten Befragung, dass sie die bereits bei T1 angesprochenen Anforderungen der sozialen Konzentration auf die Wohngruppe weiterhin ablehnte und die aus ihrer Sicht sinnlosen, unflexibel gehandhabten und nicht nachvollziehbaren Regeln der Wohngruppe zunehmend missachtete. Zusätzlich kam hinzu, „dass die auch sagten [die Betreuer*innen; Anm. d. Verf.], dass ich die gewisse Reife dafür habe, dass ich in ein betreutes Wohnen könnte" (Int. T2, Abs. 11). Saskia zog deshalb im Alter von 16 Jahren in ein betreutes Einzelwohnen, in welchem sie jedoch nur ein halbes Jahr verblieb, im Anschluss wiederum für ein halbes Jahr bei ihrer Mutter lebte und letztendlich wieder in den Haushalt ihres Vaters und dessen Lebensgefährtin einzog.

In der retrospektiven Sicht auf die Heimerziehung war in der zweiten Befragung vor allem eine Negativerfahrung in Bezug auf die Mädchenwohngruppe für sie zentral: Im Wohnheim grenzte ihr Zimmer an das Dienstzimmer, sodass sie Übergabesituationen mithören konnte.

„Die haben negativ viel über die Mädchen da geredet. Oder auch über mich. Ja teilweise fand ich auch, wars schon lästern “ (Int. T2, Abs. 41). Dieses Handeln der Betreuerinnen beschrieb Saskia als große Kränkung, die dadurch verstärkt wurde, dass „sobald die aus dem Dienstzimmer rauskamen und dich gesehen haben, haben die einfach wieder so eine Mimik aufgesetzt. [...] So einfach so totales Schauspiel und eine Maske einfach. [D]as war sehr enttäuschend fand ich" (Int. T2, Abs. 41).

Offenbar führte das Verhalten der Betreuer*innen dazu, dass der Heimerziehungskontext insgesamt negativ besetzt war, sodass sie nahezu alle sozialen Kontakte zu den Beteiligten der Heimerziehung abgebrochen hatte: „Ja mit einem Betreuer hab ich noch Kontakt aus der Wohngruppe und auch einer Mitbewohnerin. Aber so aus der Mädchenwohngruppe wollte ich auch

\footnotetext{
${ }^{5}$ Saskia Int. T2, Abs. 41.
} 
nicht, auf gar keinen Fall. Das wollte ich nicht" (Int. T2, Abs. 57).

\section{3 "So wie es jetzt ist, soll es nicht weiter ge- hen. ${ }^{\circ} 6$ - Die dritte Befragung}

Zwei Jahre später fand das dritte Interview mit Saskia statt. Saskia war zu diesem Zeitpunkt volljährig, hatte die Schulzeit mit der Fachhochschulreife beendet und eine kaufmännische Ausbildung begonnen. Die Wohnumstände waren unverändert. Sie lebte weiterhin im Haushalt ihres Vaters „und es ist alles gut. Ich möchte hier gerne noch ein bisschen wohnen bleiben. Ist so nett hier" (Int. T3, Abs. 5).

Saskia berichtete, dass sich ihre Lebensumstände seit dem Verlassen der Jugendhilfe in den zwei zurückliegenden Jahren sehr positiv verändert hatten: „[A]lso wirklich, also hat sich wirklich um 360 gewendet" (Int. T3, Abs. 13). Als Wendepunkt sah sie die Unterbringung im betreuten Einzelwohnen. In dieser Zeit war sie nahezu auf sich allein gestellt - ohne Kontakt zu ihrer Familie und mit reduzierter Unterstützung der Fachkräfte. In dieser Phase „dann war ich echt so alleine, dass ich mir dachte, so ja keinen Kontakt mehr so zur Familie und also, das war dann echt so ein bisschen einsam und traurig. [D]eswegen habe ich dann gedacht, nein, dann musste jetzt einfach in den sauren Apfel beißen und musst dich mit denen wieder vertragen, [...] weil: So, wie es jetzt ist, soll es nicht weiter gehen" (Int. T3, Abs. 39).

Nach mehrfachem Drängen erlaubte Saskias Mutter ihr den Einzug in ihren Haushalt. Das Zusammenleben der beiden war jedoch aufgrund von Auseinandersetzungen erneut nur von kurzer Dauer. Daher zog Saskia nach sechs Monaten in den Haushalt des Vaters. Die Versöhnung mit ihren Familienangehörigen hielt nun an, da „ich älter geworden bin und meine Fehler eingesehen habe" (Int. T3, Abs. 13). Sie bezog sich damit auf die Zeit zwischen erster und zweiter Unterbringung, in der sie aufgrund von Streitigkeiten mit der neuen Lebensgefährtin ihres $\mathrm{Va}$ ters nicht in deren Haushalt verbleiben konnte.
Retrospektiv beschrieb sie diese Zeit als „kritische Phase" (Int. T3, Abs. 19), in der einerseits ihr Autonomiebestreben mit den aufgestellten Regeln der Stiefmutter kollidierte, andererseits „waren auch von ihrer Seite, es waren auch genug Fehler dabei, das hat sie halt eingesehen" (Int. T3, Abs. 19).

Der vollzogene Klärungsprozess gab Saskia neuen Mut:

"[ $[\cup]$ nd weil man sich so gut wieder verstanden hat, also meine Eltern, ich und meine Geschwister und dass das Umfeld auch wieder so richtig schön passte, das hat mir, glaube ich, auch so echt den Ansporn nochmal gegeben, jetzt wirklich durchzustarten und jetzt wirklich was zu machen. [A]lso da habe ich auch gar nicht mehr an früher oder so gedacht und dann bin ich einfach jetzt so in der Gegenwart gelandet und das war auch richtig gut." (Int. T3, Abs. 37)

Saskia war zu diesem Zeitpunkt froh, nicht mehr in einer stationären Einrichtung zu leben. Sie berichtet: „Ich glaube auch nicht, dass ich, wenn ich da weiter gewohnt hätte, dass ich heute das so geschafft hätte. Auf keinen Fall, glaube ich gar nicht dran" (Int. T3, Abs. 108). Retrospektiv schaute sie auf die Zeit in den beiden vollstationären Wohngruppen eher negativ zurück. Über die Zeit in der Mädchenwohngruppe sagte sie: "Ich glaube das war das Jahr, was ich am schlimmsten im Heim fand. [...] Ich hatte da immer das Gefühl, die waren nicht ehrlich“ (Int. T3, Abs. 31). Auch die erste Unterbringung sah sie kritisch: „[D]a fehlte so ein bisschen die Unterstützung so auch von den Erziehern fand ich so, dass die da mit einem wirklich mal richtig reden" (Int.T3, Abs. 88).

\section{4 "Dass ich so ganz alleine auf mich gestellt war, das fand ich halt nicht so cool. ${ }^{4}$ - Die vierte Befragung}

Bei der vierten Befragung war Saskia 22 Jahre alt. Sie hatte ihre kaufmännische Ausbildung beendet und ein Jahr in ihrem Beruf gearbeitet, um sich eine finanzielle Rücklage aufzubauen. Da ihr der Beruf jedoch zunehmend missfiel, begann

\footnotetext{
${ }^{6}$ Saskia Int. T3, Abs. 39.

7 Saskia Int. T4, Abs. 51.
} 
6.5 „Das war nie so, dass ich einen Erzieher getroffen hätte, der ist dabei, weil inn der Mensch interessiert [...]." ${ }^{8}$ - Die fünfte Befragung

Gegen Ende des Untersuchungszeitraums zum Zeitpunkt der vorerst letzten Befragung war Saskia 25 Jahre alt und stand kurz vor dem Ende ihres Bachelorstudiums. Sie wohnte allein, bezog BAföG-Leistungen, arbeitete in einem Minijob und erwog die Aufnahme eines Masterstudiums.

Saskia befand sich in einer festen Partnerschaft mit dem Wunsch nach einer familiären Zukunft. Sie reiste viel in andere Länder, nahm gesellschaftliche und kulturelle Angebote wahr und pflegte vor allem zur Familie ihres Bruders und dessen Kindern einen engen Kontakt. Ihre Zukunftsperspektive beschrieb sie wie folgt:

„Ich möchte ein Haus haben, seit fünf Jahren verheiratet sein. [...] Und auf das erste Kind warten in meinem Garten, mit kleinen Weinträubchen auf dem Tisch. [...] Mein Ziel ist eigentlich nur glücklich zu bleiben. Ich bin glücklich und möchte glücklich bleiben, das ist mein einziges Ziel." (Int. T5, Abs. 259)

Saskias retrospektive Sicht auf die Heimerziehung war bei der fünften Befragung fast ausschließlich negativ. In Erinnerung waren Saskia die Versprechungen aller am Hilfeprozess Beteiligten (Eltern, Mitarbeiter*innen aus dem Jugendamt und Wohnheim) beim Einzug in die erste Wohngruppe geblieben.

Insgesamt sah Saskia die stationäre Jugendhilfe retrospektiv als wenig hilfreich für ihr bisheriges Leben an. Grundsätzlich nahm sie jedoch Unterstützungspotential wahr, sofern sich die Betreuungspersonen angemessen verhielten. Dies konkretisierte sie als Versuch seitens der Fachkräfte, „das Kind nicht als Objekt zu sehen, sondern ein Stück weit als Freund. Weil wenn man es als Objekt sieht, dann ist es nur Arbeit. Und da redet man einfach von Menschen und Lebewesen und die kann man nicht behandeln wie Objekte und irgendwo abstellen“ (Int. T4, Abs. 89).
„Das wurde mir gesagt, von Anfang an ein Jahr. Und dann ist alles safe und dann kann ich hingehen, wo ich möchte. Ja, war natürlich nicht so. Haben mich voll verarscht. [...] Die haben mir gesagt, wir kriegen das in einem Jahr hin. Ja war nichts mit einem Jahr, am Ende waren das vier." (Int. T5, Abs. 153-155)

Saskia ging im fünften Interview das erste Mal genauer auf das Ende der ersten Heimunterbringung ein. Sie erläuterte, dass es nach vier Jahren „immer noch keine Aussicht auf das Ende gab, [deshalb] musste ich ja selber handeln. Hätte ich nie gedacht" (Int. T5, Abs. 156). Sie beschrieb sich selbst als „so ein richtiges Ausbruchkind.

\footnotetext{
${ }^{8}$ Saskia Int. T5, Abs. 143.
} 
Habe alles schön bis zu den Grenzen, über die Grenzen strapaziert und mich mit allen da angelegt und die dann auch noch verarscht einfach, weil die wollten mich verarschen und das habe ich dann nicht eingesehen" (Int. T5, Abs. 161). Letztendlich führte dieses Verhalten zum Abbruch der Maßnahme und nötigte den Vater, sie bei sich aufzunehmen.

Retrospektiv beschrieb Saskia vor allem das Verhalten der Fachkräfte während der zweiten Unterbringung im Mädchenwohnheim:

„Das war nie so, dass ich einen Erzieher getroffen hätte, der ist dabei, weil inn der Mensch interessiert oder die Probleme oder so. Ja es war immer so, oder so habe ich es kennengelernt auch davor in dem Heim, ja ich habe heute Schicht von 08.00 bis 16.00 Uhr und danach geh ich nachhause. Was um 16.01 Uhr passiert, ist mir scheißegal." (Int. T5, Abs. 143)

\section{ANALYTISCHE ERGEBNISDARSTELLUNG}

Viele Menschen und doch allein? Saskias biografischer Verlauf ist durch zahlreiche Abbrüche, Wechsel und (soziale) Unbeständigkeiten geprägt. Aus der Vielzahl an Themen und Anknüpfungspunkten emergieren aus der Falldarstellung aus sozialisationstheoretischer Perspektive drei für den biografischen Verlauf zentrale Dimensionen, anhand derer (persönliches) Wachsen in und Gestalten von gemeinschaftlichen Prozessen sichtbar werden: Wohnen, soziale Beziehungen und beruflicher Werdegang. Die folgende Rekonstruktion der Sozialisationsprozesse ist entlang dieser analytischen Dimensionen gegliedert. Im Material treten sie jedoch in ihrer Verwobenheit miteinander in Erscheinung.

\section{1 "Und ich wollte eigentlich immer nur nach Hause." ${ }^{\prime 9}$ - Die Analysedimension Wohnen}

Der Wohn- und Lebensort als Basis für persönliche Entwicklungen ist bei Saskia aufgrund vieler Wechsel als instabil zu bezeichnen. Durch Umbrüche, Konflikte und stetig wechselnde Bezugspersonen musste sich Saskia fortwährend in neue Kontexte und interaktionale Bezugnahmen einfinden. Dadurch wurde Saskia früh vor die
Herausforderung gestellt, ihre situativen Lebensbezüge wiederholt (neu) auszuhandeln und zu gestalten. Dabei wurde sie mit Anforderungen und Regeln konfrontiert, die sie für sinnlos und unangemessen hielt (s. T1: „soziale Konzentration auf die Wohngruppe“). Zudem erfuhr sie stetig, dass sie sowohl in den familiären Haushalten als auch in verschiedenen Wohngruppen scheinbar nicht richtig aufgehoben war. Durch die Aufschichtung der eigenen Erfahrungsbiografie mit negativen Erlebnissen generierte Saskia zunehmend Misstrauen gegenüber erwachsenen Bezugspersonen (s. T1: „Vater, Mutter, Lebensgefährtin“; T2: „Betreuer*innen“).

Die wechselnden Wohnbezüge und Bezugspersonen verstärkten einerseits möglicherweise eine negative Sichtweise auf sich selbst, andererseits führten sie zu differenten Handlungsbefähigungen: Durch erlebte Vertrauensbrüche und den Mangel an verlässlichen Bezugspersonen war Saskia früh auf Selbstständigkeit angewiesen. Sie übernahm Selbstverantwortung für ihr Leben und ihre Situation, sorgte für sich und versuchte Lebensentscheidungen aktiv mitzugestalten (s. T3: „Aufnahme in den Haushalt der Mutter und anschließend in den Haushalt des Vaters"). Daraus resultiert auch, dass Saskia früh lernte, (nicht immer sozial erwünschte) Entscheidungen zu treffen und umzusetzen, die sich wiederrum auf die Zeit in Heimunterbringung auswirkten (s. T5: „Ausbruchkind“).

Während dieser Entwicklungsprozesse nahm Saskia wiederholt Desinteresse und unauthentische Verhaltensweisen der pädagogischen Fachkräfte wahr, sodass ihr Wohnumfeld in den verschiedenen Settings in ihren Augen von Unehrlichkeit und emotionaler Distanz gekennzeichnet war (s. T2: „Lästern und Schauspiel"). Saskia konnte nur sich selbst vertrauen. Der Rückbezug auf sich selbst und auf persönliche Stärken sowie eine in sich selbst verankerte Wertigkeit ermöglichten Saskia offenbar, mit differenten Handlungsanforderungen und -notwendigkeiten produktiv umzugehen. Sie schaffte es beispielsweise auf unterschiedliche Weise, den negativ erlebten Aufenthalten zu entfliehen, um anschließend von sich aus Klärungsprozesse

\footnotetext{
9 Saskia Int. T4, Abs. 130.
} 
zur Wiederaufnahme in familiäre Haushalte anzustoßen.

Alles in allem scheint diese Entwicklung von Persönlichkeit und Handlungsbefähigung durch den Bereich des Wohnens bei Saskia (teilweise) unbewusst verlaufen zu sein. Sie konnte zwar den Erwerb handlungspraktischer Fertigkeiten als besondere Entwicklungsprozesse in der stationären Heimunterbringung benennen (s. T1: „Leben in die eigene Hand genommen“), jedoch fiel ihr die Erklärung für den aus ihrer Perspektive positiven poststationären biografischen Verlauf schwer. Sie konnte ihre positiven Entwicklungsprozesse aus den vornehmlich negativen Erlebnissen und Erfahrungen der wechselnden Unterbringungsformen und ihren damit zusammenhängenden Handlungsbezügen reflexiv nicht einholen. Sie hatte zwar eine Idee, dass die Erfahrung des Allein- und Verlassenseins im Einzelwohnen ein Wendepunkt gewesen sein könnte, jedoch blieb eine genaue Beschreibung aus. Die Erlebnisse schienen durch Enttäuschungen verdeckt zu sein und bahnten sich ihren Weg in die Alltagspraktiken von Saskia vor allem in der Zeit nach der stationären Heimunterbringung.

\section{2 „Hauptsache irgendwie so ein Stückweit Fa- milie." ${ }^{10}$ - Die Analysedimension soziale Bezie- hungen}

Unterschiedliche soziale Beziehungen prägten Saskias biografischen Verlauf. Die Beziehung zwischen Saskia und ihren Eltern kann als ambivalent interpretiert werden. Bis zur Trennung ihrer Eltern erlebte Saskia das Familienleben als harmonisch. Erst durch das reduzierte Interesse ihres Vaters, die Alkoholerkrankung ihrer Mutter und die daraus resultierende Heimunterbringung wurde die Beziehung zu Saskias Eltern krisenhaft. Denn aufgrund der scheinbar mangelnden Partizipation am Prozess der Unterbringung fühlte sich Saskia fremdbestimmt und abgeschoben (s. T1: „Sie wollten mich nicht mehr.").

Einerseits besaß Saskia den Wunsch nach einem liebevollen und engen Kontakt zu ihren Eltern, den sie durch die wiederholten Versuche des Einzugs in den mütterlichen bzw. väterlichen
Haushalt verdeutlichte. Anderseits bemerkte Saskia vermehrt die Begrenztheit der Möglichkeiten des Zusammenlebens mit ihren Eltern. Sie wurde wiederholt enttäuscht und führte den ambivalenten Kontakt zu ihren Eltern während der Heimunterbringung zunächst fort, um im Anschluss nach einem durch sie initiierten Klärungsprozess einen für sie adäquaten Kontakt zu leben (s. T3: „Klärungsprozess“).

Während der Zeit der Jugendhilfe vollzogen sich die sozialen Beziehungen zu den wechselnden Betreuer*innen ebenfalls krisenhaft. Sowohl im stationären Bereich als auch in der ambulanten Betreuung fühlte sich Saskia nicht angemessen behandelt. In der Folge zog sich Saskia schon früh aus sozialen Heimbezügen zurück und grenzte sich gegenüber dem Heimkontext ab (s. T5: „Verarschen“). Als Folge forderten die pädagogischen Fachkräfte ein verstärktes Einbringen in der Wohngruppe, während Saskia parallel dem für sie engen Kontext entfloh. Saskia wurde auf diese Weise entgegen ihrer Bestrebungen in den Kontext Heimunterbringung gedrängt. Sozialisationstheoretisch erlebte Saskia während der Jugendhilfezeit eine asymmetrische wechselseitige Bezugnahme zu erwachsenen Menschen (Fachkräften und Eltern), die individuelles Diskrepanzerleben hervorbrachte. Durch die zunehmende Enttäuschung fühlte sich Saskia scheinbar mehr und mehr im Stich gelassen, grenzte sich gegenüber sozialen Beziehungen im Heimkontext ab und gestaltete ihr Leben möglichst eigenverantwortlich und selbstständig.

Saskia erzählte wenig über ihre Peerkontakte während der Heimunterbringung. Sie pflegt noch rudimentären Kontakt zu einer Mitbewohnerin zu den anderen Gleichaltrigen sind die Beziehungen abgebrochen. Das spricht aus Sicht von Saskia für überwiegend temporäre Bezugnahmen zu Mitbewohner*innen in der Jugendhilfe, die nur übergangsweise Bedeutung hatten (s. T4: „Man hat halt die Zeit zusammen verbracht.").

Saskias Zukunftsvision fokussiert vor allem verlässliche Strukturen und Beziehungen, die sie selbst nicht erleben konnte - inklusive verlässli-

\footnotetext{
10 Saskia Int. T4, Abs. 130.
} 
cher Partnerschaft, eigenem Haus und Weintrauben auf dem Tisch als Zeichen sicherer Versorgung. Saskia wünscht sich Nähe und Geborgenheit in ihren familiären Interaktionen. Die gemeinsame Lebensführung mit ihren Familienangehörigen ist in ihrer Vision durch Anerkennung, Liebe, Verlässlichkeit und gesicherte Versorgung gekennzeichnet. Aspekte, die sie scheinbar nur in geringem Maße während ihrer Heimzeit erleben durfte.

\section{3 „Ich glaube auch nicht, dass ich, wenn ich da weiter gewohnt hätte, dass ich heute das so ge- schafft hätte." ${ }^{11}$ - Die Analysedimension berufli- cher Werdegang}

Zu den Themen Ausbildung und Arbeit äußerte sich Saskia vor allem nach der Zeit der stationären Unterbringung. Die neu gefundene Passung der Wohn- und Lebensumstände hatte Saskia angespornt, sich beruflich weiterzuentwickeln. In ihren Narrationen ist zu erkennen, dass sich Saskia durch die Befriedung ihrer Lebens- und Handlungskontexte befähigt und motiviert sah, eigene berufliche Ziele zu entwickeln und zu verfolgen (s. T3: „Durchstarten“): Eingebettet in die familiären Bezüge väterlicherseits konstruierte Saskia nach dem Klärungsprozess eine Form gemeinsamer Lebensführung, die parallel Gemeinschaft und Individualität hervorbrachte. Auf diese Weise schafften die Beteiligten gemeinsam die Grundlage für Saskias berufliche Entfaltung.

Sozialisationstheoretisch emergierten aus der wechselseitigen Bezugnahme innerhalb der Familie individuelle Prozesse von Handlungsbefähigung und Individualgenese. Erst nach der Herstellung verlässlicher und passgenauer familiärer Beziehungen schien sich Saskia beruflich entwickeln zu können. Sie konnte die entwickelten Kompetenzen aus ihrer Erfahrungsbiografie für ihre eigene Ausbildung, ihr Studium und ihre Berufspraxis einsetzen - und das mit Erfolg. Sie erwarb einerseits gesellschaftlich anerkannte Bildungszertifikate und behauptete sich andererseits in der beruflichen Praxis. Außerdem hatte sie Zukunftsvisionen entwickelt, die ihre beruflichen Perspektiven noch erweitern (s. T5: „Masterstudium").
Es ist festzustellen, dass sich Saskias beruflicher Werdegang aus eigenem Antrieb entwickelte. Die Kompetenzen und Befähigungen dazu gingen aus einer ko-konstruierten sozialen Familienpraxis nach der stationären Heimunterbringung hervor. Dies lässt vermuten, dass es zur Zielerreichung einer emotionalen und persönlichen Zuwendung bedurfte, die Saskia während der Heimunterbringung für sich nicht erfahren hatte. Offenbar aus diesem Grund konnte Saskia erst nach der stationären Jugendhilfe ihre beruflichen Ziele verfolgen - aus sicheren und emotional gefestigten Bezügen heraus.

\section{RESÜMEE}

Im biografischen Verlauf von Saskia werden vielfältige Sozialisationseffekte während und nach den verschiedenen Unterbringungen sichtbar. Im Einzelfall zeigen sich Muster in den Dimensionen Wohnen, soziale Beziehungen und beruflicher Werdegang, die Rückschlüsse auf Aspekte zulassen, die bei der Ausgestaltung stationärer Jugendhilfeangebote für die Adressat*innen von Bedeutung sind.

Auch wenn die Einzelfallanalyse keinen Anspruch auf Repräsentativität erhebt, ist festzustellen, dass Sozialisation in und durch Heimerziehung als "kritisches Lebensereignis" (Lambers 1996) für die betroffenen jungen Menschen (und ihre Bezugspersonen) besonders herausfordernd und zeitlich beständig ist. Die Persönlichkeitsentwicklung und Verselbständigung, folglich das Erlernen von alltagspraktischen Fertigkeiten, die Fähigkeit in verschiedenen Lebenssituationen angemessen zu agieren und die Entwicklung emotionaler Stabilität - kurz die Handlungsbefähigung - verläuft unter erschwerten Bedingungen. Zudem vollzieht sich das Hineinwachsen in gesellschaftliche Bezüge aus vergleichsweise unsicheren und häufig wechselnden Strukturen. Besonders deutlich zeigt sich das am Fall von Saskia in den dargestellten Ambivalenzen und Schwierigkeiten in Bezug auf divergierende Familienkonstellationen (Analysedimension soziale Beziehungen) und Wohnsettings (Analysedimension Wohnen). Denn die unterschiedlichen

\footnotetext{
11 Saskia Int. T3, Abs. 108.
} 
Lebenskontexte erfordern vielfältige Anpassungsprozesse bei paralleler impliziter Botschaft eines nicht Erwünscht- und Richtigseins. Das wiederrum erschwert eine eigene, individuellkongruente Persönlichkeitsentwicklung.

Der Fall Saskia macht einerseits deutlich, dass eine geringe Stabilität des Wohnumfeldes, ein Mangel an tragfähigen sozialen Beziehungen, ein unauthentisches Handeln (insbesondere von sozialpädagogischen Akteur*innen der stationären Erziehungshilfe) sowie eine geringe Partizipation und Förderung der individuellen Bedürfnisse die Integration in soziale Bezüge und die Arbeitswelt hemmen (Analysedimension beruflicher Werdegang). Anderseits belegt der Fall Saskia, dass die instabilen Sozialisationskontexte zu ausgeprägter Handlungsfähigkeit führen können. Saskia konnte die Erlebnisse und Erfahrungen positiv integrieren und persönliche Stärke entwickeln. Die Gelingensfaktoren der aktiven Lebensgestaltung von Saskia könnten den Bedingungen der Heimerziehung zugeschrieben werden, auf individuellen protektiven Ressourcen basieren oder Ergebnisse glücklicher Zufälle sein. Eindeutige Schlussfolgerungen erscheinen nicht möglich, aber sicher ist: Aus Saskias biografischem Verlauf emergierten trotz der erschwerten Kontextbedingungen vielfältige Sozialisationseffekte.

In der längsschnittlichen Betrachtung des Falls fällt auf, dass für Saskia auch lange nach der Jugendhilfe das (sozialpädagogische) Handeln der Fachkräfte von zentraler Bedeutung ist. Saskia sieht in den Fachkräften sogar den entscheidenden Faktor für gute Heimerziehung. Folglich sind die sozialpädagogischen Fachkräfte maßgeblich daran beteiligt, die schwierigen Bedingungen im institutionellen Kontext Heimerziehung für untergebrachte junge Menschen zu gestalten. Der Fall Saskia zeigt insbesondere, dass die pädagogischen Fachkräfte die erschwerten Sozialisationsprozesse durch ein tragfähiges Beziehungsangebot (Wieland et al. 1992; Finkel 2004) sowie durch die Förderung heimexterner (sozialer) Ressourcen (Macsenaere/Esser 2015) unterstützen können. Innerhalb der Münsteraner Längsschnittstudie wird auch in anderen Fällen die hier dargestellte subjektiv hohe Relevanz der sozialpädagogischen Fachkräfte für die Adressat*innen der Heimerziehung sichtbar. Sowohl
Negativerfahrungen als auch positiv wahrgenommenes Verhalten blieben langfristig in Erinnerung und waren zudem meistens mit der Bewertung der Hilfemaßnahme gekoppelt.

Ausgehend von unserer Analyse ergeben sich folgende Schlussfolgerungen: Professionelles Handeln sozialpädagogischer Fachkräfte sollte stetig (selbst-)reflexiv erfolgen. Die Reflexionsprozesse umfassen die prozesshafte Evaluation der biografischen Passung (Finkel 2004) und des Verlaufs der Maßnahme entlang der Bedürfnisse der jungen Menschen, um das pädagogische Handeln entsprechend auszurichten. Eine daraus resultierende professionell-sichere Beziehung zwischen Adressat*innen und Fachkräften kann eine instabile und ambivalente Beziehung zu Elternteilen ergänzen, sodass eine durchaus anspruchsvolle Elternarbeit auch im Kontext stationärer Heimunterbringung nicht ausgeschlossen ist. Denn der Fall Saskia zeigt: Für viele junge Menschen verschwinden die Bezüge zu ihren Eltern, der Wunsch nach Familie sowie nach (alten) sozialen Kontakten außerhalb der Einrichtung nicht. Die sozialen Beziehungen sind vielmehr Grundlage, Bestandteil und Ergebnis von individueller Entwicklung und gesellschaftlichen Partizipations- und Gestaltungsprozessen.

Das führt dazu, dass Sozialisationsprozesse während der Heimerziehung sowohl eine gegenwärtige biografische Passung als auch zukunftsorientierte Ziele zu berücksichtigen haben - sie sind als situativ bedingt im zeitlichen biografischen Verlauf zu sehen. Auf diese Weise gerät die Gestaltung von Beziehungen, Netzwerken und Außenbezügen nicht nur im Care-LeavingProzess in den Blick, sondern schon während der gesamten Heimunterbringung. Sind diese Voraussetzungen erfüllt, ermöglicht das Aufwachsen in Heimerziehung vielfältige Sozialisationseffekte.

\section{LITERATUR}

Abels, Heinz (2015): Der Beitrag der Soziologie zur Sozialisationsforschung. In: Hurrelmann, Klaus/Bauer, Ullrich/Grundmann, Matthias/Walper, Sabine (Hrsg.): Handbuch Sozialisationsforschung. 8. Auflage. Weinheim/Basel: Beltz, 5079. 
Ader, Sabine/Schrapper, Christian (Hrsg.) (2020): Sozialpädagogische Diagnostik und Fallverstehen in der Jugendhilfe. München: Ernst Reinhardt.

Bauer, Ullrich (2012): Das sozialisationstheoretische Paradigma. In: Bauer, Ullrich/Bittlingmayer, Uwe H./Scherr, Albert (Hrsg.): Handbuch Bildungs- und Erziehungssoziologie. Wiesbaden: Springer VS, 473-490.

Clausen, John Adam (1968): Socialization and Society. Boston: Little, Brown \& Co.

Fendrich, Sandra/Pothmann, Jens/Tabel, Agathe (2018): Monitor Hilfen zur Erziehung 2018. Dortmund: Eigenverlag Forschungsverbund DII/TU Dortmund.

Franz, Julia/Unterkofler, Ursula (Hrsg.) (2021): Forschungsethik in der Sozialen Arbeit. Prinzipien und Erfahrungen. Opladen/Berlin/Toronto: Verlag Barbara Budrich.

Finkel, Margarete (2004): Selbständigkeit und etwas Glück. Einflüsse öffentlicher Erziehung auf die biographischen Perspektiven junger Frauen. Weinheim: Juventa.

Gehres, Walter (1997): Das zweite Zuhause. Institutionelle Einflüsse, Lebensgeschichte und Persönlichkeitsentwicklung von dreißig ehemaligen Heimkindern. Opladen: Leske + Budrich.

Grundmann, Matthias (2006): Sozialisation. Skizze einer allgemeinen Theorie. Konstanz: UVK.

Hansbauer, Peter/Kress, Laura (2012): Übergänge in die Zeit nach dem Heim. Ergebnisse aus einem Projekt mit ehemaligen Jugendlichen aus den Erziehungshilfen. Münster: Ohne Verlag.

Lambers, Helmut (1996): Heimerziehung als kritisches Lebensereignis. Münster: Votum.

Lamnek, Siegfried/Krell, Claudia (2016): Qualitative Sozialforschung. 6. Auflage. Weinheim/Basel: Beltz.
Macsenaere, Michael/Esser, Klaus (2015): Was wirkt in der Erziehungshilfe? Wirkfaktoren in der Heimerziehung und anderen Hilfearten. 2. Auflage. München: Ernst Reinhardt.

Normann, Edina (2003): Erziehungshilfen in biografischen Reflexionen. Heimkinder erinnern sich. Weinheim: Beltz Votum.

Nüsken, Dirk/Böttcher, Wolfgang (2018): Was leisten die Erziehungshilfen? Eine einführende Übersicht zu Studien und Evaluationen der HzE. Weinheim: Beltz Juventa.

Parchow, Alexander (2021): Lebenschance Heimerziehung? Der Einfluss von stationärer Erziehungshilfe auf die Biografie junger Menschen. In: Brockmann, Lilo/Hack, Carmen/Pomykaj, Anna/Böttcher, Wolfgang (Hrsg.): Soziale Ungleichheit im Bildungs- und Sozialwesen. Reproduktion und Legitimierung. Weinheim/Basel: Beltz Juventa, 114-129.

Rätz-Heinisch, Regina (2005): Gelingende Jugendhilfe bei „aussichtslosen Fällen“! Biographische Rekonstruktionen von Lebensgeschichten junger Menschen. Würzburg: Ergon.

Sierwald, Wolfgang/Weinhandl, Kathrin/Salzburger, Veronika/Straus, Florian (2017): Wie Care Leaver den Weg in die Selbstständigkeit erleben. Erste Ergebnisse aus der SOS-Längsschnittstudie zur Handlungsbefähigung. In: Unsere Jugend, 69 (1), 10-19.

Strahl, Benjamin (2020): Heimerziehungsforschung in Deutschland. Eine Expertise für das Zukunftsforum Heimerziehung. Frankfurt am Main: IGfH-Eigenverlag.

Strauss, Anselm Leonard/Corbin, Juliet $M$. (1996): Grounded Theory. Grundlagen Qualitativer Sozialforschung. Weinheim: Beltz.

Wieland, Norbert/Marquard, Uschi/Panhorst, Hermann/Schlotmann, Hans-Otto (1992): Ein Zuhause - kein Zuhause. Lebenserfahrungen und -entwürfe heimentlassener junger Erwachsener. Freiburg im Breisgau: Lambertus. 


\section{Zu den Autoren}

Alexander Parchow ist Sozialarbeiter, M. A. Jugendhilfe - Konzeptionsentwicklung und Organisationsgestaltung und wissenschaftlicher Mitarbeiter an der Fachhochschule Münster am Fachbereich Sozialwesen mit dem Arbeits- und Forschungsschwerpunkt erzieherische Hilfen. Er promoviert am Institut Erziehungswissenschaften der Westfälischen Wilhelms-Universität Münster zu dem Thema der Wirkungen, Folgen und Bedeutung stationärer Heimerziehung im Leben junger Menschen.

Tim Middendorf ist Dipl.-Sozialarbeiter, Supervisor (M. A.) und wissenschaftlicher Mitarbeiter an der Katholischen Hochschule Nordrhein-Westfalen in Münster. Er promovierte am Institut für Soziologie der Westfälischen Wilhelms-Universität Münster am Lehrstuhl für Sozialisationsforschung zum Thema „Professionalisierung im Studium der Sozialen Arbeit. Eine sozialisationstheoretische Perspektive auf Ausbildungssupervision".

\section{Kontakt}

\section{Alexander Parchow}

Fachhochschule Münster, Fachbereich Sozialwesen

Hüfferstraße 27

D-48149 Münster

E-Mail: alexander.parchow@fh-muenster.de

URL: https://www.fh-muenster.de/sw/personen/Wissenschaftliche_Mitarbeiterlnnen.php?pld=8976\&orga $=10 \& m \_l i s t \_i d=0 \& p \_l i s t \_i d=20$

Dr. Tim Middendorf

Katholische Hochschule NRW, Abteilung Münster

Piusallee 89

D-48147 Münster

E-Mail: t.middendorf@katho-nrw.de

URL: https://www.katho-nrw.de/muenster/studium-lehre/lehrende/wissenschaftlich-mitarbeitende/middendorf-tim-dr-phil/ 